\title{
Neutron star structure: what we learn from their masses and radii
}

\section{Feryal Özel}

University of Arizona, United States

email: fozel@email.arizona.edu

\begin{abstract}
Neutron stars possess the densest matter and strongest gravitational fields that are accessible to observations. In this talk, I will discuss how precise measurements of neutron star radii, masses, and spins not only open a window onto the poorly known neutron star interior but can also be used to probe their formation mechanism, their recycling to millisecond periods, and their connection to the formation of low-mass black holes.
\end{abstract}

